\title{
Corruption naturelle et corruption civilisationnelle dans le Theophrastus Redivivus (1659)
}

Jean-Pierre Cavaillé

\section{(2) OpenEdition \\ 1 Journals}

Édition électronique

URL : http://journals.openedition.org/edl/925

DOI : $10.4000 /$ edl. 925

ISSN : 2296-5084

Éditeur

Université de Lausanne

\section{Édition imprimée}

Date de publication : 15 décembre 2015

ISBN : 978-2-940331-47-5

ISSN : 0014-2026

\section{Référence électronique}




\section{CORRUPTION NATURELLE ET CORRUPTION CIVILISATIONNELLE DANS LE THEOPHRASTUS REDIVIVUS (1659)}

L'article formule et affronte un paradoxe qui se trouve au cœur du traité anonyme intitulé Theophrastus redivivus, mais aussi, au-delà, de ce qu'il est convenu d'appeler le libertinisme ou le naturalisme libertin. Cet ouvrage, dont l'objectif est la mise en œuvre d'un projet philosophique d'émancipation des erreurs et mensonges des religions révélées, réfute en effet la conception chrétienne de la corruption, fondée sur le concept de péché et sur le principe théologique du péché originel source de toute corruption, pour revenir à une conception strictement physiciste de la corruption, complémentaire de la génération qui sont les deux principes naturels auxquels sont soumis les êtres naturels sublunaires. Mais cet affranchissement philosophique, véritable retour à la nature, fait apparaître l'ensemble de la société humaine régie par l'illusion religieuse, et structurée par des lois et des préceptes qui fondent la servitude et l'inégalité entre les hommes, comme un état de corruption, d'altération radicale eu égard à la loi naturelle, que le sage considère comme sa seule règle d'action. Il y a ainsi une tentation perfectiste chez le sage libertin, seul esprit affranchi et incorrompu, dans un monde humain radicalement corrompu; une corruption morale et intellectuelle dont le seul recours à l'histoire naturelle a bien du mal à rendre compte.

Dans les lignes qui suivent, nous voudrions mettre en lumière un paradoxe qui nous paraît se trouver au cœur du Theophrastus redivivus, manuscrit clandestin, anonyme, achevé en 1659. Ce traité se présente comme une mise en ordre encyclopédique et produit à la fois une interprétation radicale de motifs philosophiques anciens (aristotéliciens, épicuriens, sceptiques, stoïciens, cyniques) et d'auteurs modernes (Machiavel, Pomponazzi, Cardan, Charron, Vanini, etc.) qui sapent les fondements des religions révélées au profit d'une vision du monde souvent (et dès l'époque) nommée naturaliste, du fait que la nature s'y 
trouve appréhendée comme l'entité englobant l'ensemble de ce qui existe. Ainsi le paradoxe que nous voulons mettre en lumière n'est-il pas propre au Theophrastus, mais se trouve au cour de cette culture philosophique qu'il est convenu de nommer "libertine», qui produit, de manière plus ou moins ouverte ou couverte, une critique foncière de la religion ramenée à ses fonctions éthiques et politiques, au profit de l'élection, réservée à l'élite des esprits forts, d'une sagesse prenant la nature comme modèle exclusif ${ }^{1}$.

\section{Le paradoxe de la corruption}

On peut tenter, en simplifiant au maximum, de formuler ce paradoxe de la manière suivante. Le projet philosophique d'émancipation consiste essentiellement à revenir à une conception strictement physiciste, naturaliste de la corruption, comme complémentaire de la génération, les deux principes auxquels sont soumis les êtres naturels sublunaires (le cadre physique et cosmologique de l'anonyme reste dans l'ensemble fidèle à la tradition aristotélicienne). Ce retour à la nature, qui est d'abord un retour aux textes anciens (d'Aristote et des aristotéliciens ainsi que de la tradition épicurienne, auxquelles s'ajoutent des influences stoïciennes, sceptiques et cyniques), implique que l'on s'affranchisse de la conception à la fois religieuse, morale et politique de la corruption comprise en premier lieu comme figmentum malum, mauvais fond de l'homme, disposition à la faute, à la transgression des lois et des préceptes prétendument institués par ces entités supranaturelles que l'on appelle, au singulier ou au pluriel, "Dieu(x)». Mais cet affranchissement philosophique fait apparaître l'ensemble de la société humaine, régie par l'illusion religieuse et structurée par des lois et des préceptes qui fondent la servitude et l'inégalité entre les hommes, comme un état de corruption,

I. Sur le Theophrastus redivivus, encore à ce jour non traduit intégralement en français, voir T. Gregory, Theophrastus redivivus; M. Rodríguez Donís, Materialismo $y$ ateismo et N. Gengoux, Le "Theophrastus redivivus" ou l'athéisme comme position philosophique à l'Age classique. Nous citons l'édition critique de G. Canziani et G. Paganini. Depuis la composition de cet article, N. Gengoux a tiré un ouvrage de sa thèse (Un athéisme philosophique à l'Age classique). Voir également H. Bah-Ostrowiecki, Le Theophrastus redivivus et N. Gencoux et P.-F. Moreau (dir.), Entre la Renaissance et les Lumières. 
d'altération radicale eu égard à la loi naturelle que le sage élit comme seule règle $\mathrm{d}$ 'action.

Dès lors l'homologie est frappante entre la conception religieuse de la corruption qui, en termes chrétiens, s'exprime à travers les notions de chute et de péché, et cette conception philosophique qui déplore la perte de la liberté et de l'égalité naturelles et constate l'altération et la dénégation de la loi de nature. Elle est, aussi bien, le parallélisme que l'on peut établir entre la figure du saint qui s’arrache à la corruption du péché et celle du sage qui s'exempte de la corruption de la civilisation humaine dénaturée. Cette homologie, qui se traduit par certains thèmes communs (notamment celui de la vanité de l'homme) est, comme nous verrons, bien loin de se laisser réduire pour autant à une identité.

\section{Démolition du dogme de la nature corrompu}

La première chose à remarquer est la capacité du Theophrastus, sur une base principalement aristotélicienne et épicurienne, à se débarrasser de la déclinaison spécifiquement chrétienne de ce que nous venons de nommer conception religieuse de la corruption. C'est qu'en régime chrétien, la corruption de la nature est elle-même érigée en dogme. La corruption en effet, dans la "fable» théologique chrétienne, est conçue comme présence active du mal, comme faute introduite dans la nature elle-même par le péché originel, en l'homme et hors de l'homme ${ }^{2}$. Cette conception proprement théologique suppose l'existence d'un Dieu créateur omnipotent et souverainement bon, comme tel absolument inaltérable et incorruptible, auteur d'une nature qu'il a créée vierge de toute corruption. Il la créa certes corruptible et savait nécessairement (sinon quel Dieu aurait-il été ?) de toute éternité qu'elle serait corrompue, ce qui ne

2. L'auteur anonyme du Theophrastus redivivus ne manque pas de produire une critique conséquente du dogme du péché originel. Voir traité III, chapitre 5, p. 500 sq. (dorénavant abrégé III, 5), mais aussi l'allusion et le très important argument en VI, I, p. 794. Nous donnerons ici la traduction des passages cités tout en renvoyant à la pagination de l'édition de Canziani et Paganini. Nous tenons à remercier N. Gengoux qui prépare une traduction française de l'ensemble du Theophrastus et qui nous a aimablement communiqué son travail. Il existe cependant déjà une traduction publiée du livre VI, réalisée par Hélène Bah-Ostrowiecki et accompagnée du texte latin. C’est elle que nous utilisons comme base lorsque nous citons ce dernier livre. 
permet d'aucune façon pourtant, en toute bonne théodicée, de le juger responsable de la corruption effective et de la présence du mal dans le monde.

Du reste, ce Dieu est aussi un Dieu rédempteur qui, par l'incarnation, l'assomption en son fils d'un corps corruptible et la mort ignominieuse sur la croix, ouvre les portes du salut, c'est-à-dire la possibilité pour l'homme d'accéder, une fois le corps abandonné à sa corruption matérielle par l'épreuve de la mort, à la béatitude de son âme immortelle, c'est-à-dire à une jouissance de Dieu délivrée de toute corruption avec la promesse d'une résurrection dans un corps glorieux, cette fois incorruptible.

C'est cette "fable» chrétienne de la nature corrompue et de la régénération accordée par la grâce du Dieu rédempteur (on essaie ici d'en présenter une version "standard", car il s'agit pour le courant de pensée que nous évoquons d'aller et de tailler à la racine, bien en deçà des querelles de la grâce et du libre-arbitre), que démolit sans appel la philosophie naturaliste du Theophrastus en revenant à la conception strictement physicienne du cycle aristotélicien des générations et des corruptions qui, dans la théologie scolastique, en particulier thomiste, était strictement contenue et limitée par le dogme créationniste. La corruptibilité des êtres naturels, dans cette perspective, attestait d'ailleurs et en quelque sorte trahissait une déficience ontologique, renvoyant, hors de la nature sensible à la cause créatrice; elle servait à construire l'un des arguments de la preuve dite cosmologique.

\section{Un Dieu incorruptible n'est rien}

L'anonyme suit un chemin exactement inverse: l'une des preuves qu'il apporte pour démontrer l'inexistence de Dieu, adaptée de Sextus Empiricus $^{3}$, est tirée de l'observation de la corruption et de la mort chez les animaux ${ }^{4}$. Il s'agit d'un argument strictement sensualiste, rude, car il consiste à prendre à la lettre certaines expressions pour désigner l'omniscience divine, mais non dénué pour autant d'efficacité.

3. Adversos mathematicos, IX, 176-179, référence donnée par C. Canziani et G. Paganini dans le très riche appareil critique de leur édition, p. 152.

4. I, 6, p. 152. 
On affirme que "Dieu voit et entend tout", or seul les animaux ont la capacité de voir et d'entendre, et Dieu ne saurait être un animal, car «il serait exposé à la corruption et à la mort; or la mort ni la corruption n'affectent Dieu; donc il n'est pas un animal». Mais s'il n'est pas un animal, il est absurde de dire qu'il entend et qu'il voit; s'il ne sent rien, Dieu ne saurait rien connaître et comprendre. Ainsi n'est-il «ni corporel, ni incorporel. Donc Dieu n'est rien, sinon une fiction ${ }^{5}$.

En fait la corruptibilité des animaux renvoie bien à un être incorruptible, mais dans la plus pure immanence: le monde même, éternel, incréé, donc inengendré et incorruptible ${ }^{6}$. Du reste, une fois en effet que l'on s'est délivré du dogme de la création, tant il en découle "de choses absurdes et impossibles», et que l'on s'est donc débarrassé de Dieu, force est de conjecturer que le monde est lui-même «nécessaire, inengendrable et incorruptible». Le monde en effet gagne son autonomie par l'absorption, mais sur un plan de pure immanence, des qualités divines d'éternité, d'inengendrabilité et d'incorruptibilité7.

\section{Le cycle naturel des générations et des corruptions (mortalité de l'homme et donc de l'âme)}

Ainsi, en ce monde sublunaire, ne rencontre-t-on que des êtres engendrés et corruptibles ${ }^{8}$, mais leurs espèces ne le sont pas, "qui s'écoulent de toute éternité». Encore que l'anonyme émette, pour les animaux dits "parfaits» qui ne peuvent se reproduire que par la semence, comme l'homme, la possibilité d'une disparition sinon totale, du moins quasi totale de l'espèce par extinction des géniteurs à l'occasion de catastrophes naturelles. Car l'homme, pour l'anonyme, ne jouit d'aucune prérogative

5. Ibid.

6. II, 1, p. 176 et sq.

7. Et l'on comprend d'emblée pourquoi d'ailleurs la solution panthéiste pouvait paraître s'imposer d'elle-même dans cette culture philosophique. Mais l'auteur du Théophrastus ne divinise pas la nature, au sens où il ne lui reconnaît aucune dimension providentielle. Il est par contre tout à fait fondé de parler à son sujet de «matérialisme». Voir N. Gengoux, Le "Theophrastus redivivus», chap. VI et VII.

8. L'auteur puise ici, bien sûr, dans le traité De generatione et corruptione d'Aristote, non sans apporter cependant sa touche. Voir la note de C. Canziani et G. Paganini, p. 176. 
par rapport aux autres animaux, au contraire, sous de nombreux aspects, il est inférieur à nombre de ses congénères.

Tout en lui, d'abord, est corruptible et périssable. L'anonyme est très attentif à démontrer que l'immortalité de l'âme relève de la fable religieuse; l'âme est inséparable du corps dont elle est la forme et se corrompt avec lui, irrémédiablement :

Il faut reconnaître que l'âme est corrompue avec le corps parce qu'elle est engendrée avec lui, en effet, tout ce qui est engendrable est aussi corruptible, et de même cesse parce qu'il a commencé [...] donc l'âme est parfaitement mortelle, et n'est d'aucune manière supérieure au corps 9 .

La condition de l'homme est ainsi, de part en part, celle d'un être corruptible, qui se dissout avec la mort dans la nature, et il est aussi absurde d'affirmer l'immortalité de l'âme que la résurrection des corps. A ce propos l'anonyme cite et commente divers versets des Psaumes et du Livre de Job (l'usage de la Bible contre le christianisme est une stratégie constante des «libertins»), lieux topiques de la thématique de la vanité de l'homme omniprésente à la même époque dans l'ensemble de l'Europe:

[...] nous ne sommes que poussière; le jour de l'homme passe comme l'herbe; il est comme la fleur des champs qui fleurit pour un peu de temps. Parce que l'esprit ne fera que passer en lui, et que l'homme ensuite ne subsistera plus, et il n'occupera son lieu comme avant" (Psaume 102, 14-15).

Et encore: "Comme une nuée se dissipe et passe sans qu'il en reste de trace, ainsi celui qui descend ad inferos ne remontera plus" (Job 7. 9), entendu par inferos, remarque justement l'anonyme, "la terre et la mort» (d'ailleurs Le Maître de Sacy, dont nous utilisons la traduction, traduit bien lui-même ad inferos par «tombeau»). Mais, poursuit-il, rien n'est plus efficace contre l'immortalité de l'âme et la résurrection des corps que ce passage très clair du même Job:

Un arbre n'est point sans espérance; quoiqu'on le coupe, il ne laisse pas de reverdir, et ses branches poussent de nouveau. Quand sa racine serait vieillie dans la terre, quand son tronc desséché serait mort dans

9. IV, 3, p. 640. 
la poussière. Il ne laissera pas de pousser aussitôt qu'il aura senti l'eau, et il se couvrira de feuilles comme lorsqu'il a été planté. Mais quand l'homme est mort une fois, dépouillé et consumé, je demande où il est? De même que les eaux se retirent de la mer, et qu'un fleuve devient à sec, ainsi quand l'homme est mort une fois, il ne ressuscitera point (Job, 14. 7-12).

Certes, le texte biblique ajoute "jusqu'à ce que le ciel soit détruit», mais il s'agit là d'une probable interpolation ajoutée par «un corrupteur», qui contredit l'affirmation précédente concernant le fait que l'homme «est mort et consumé»: le verbe "consumer», en effet, signifie l'annihilation pour ainsi dire absolue et intégrale ${ }^{10}$.

\section{L'homme est moins que l'insecte}

L'homme est donc entièrement soumis à la corruption naturelle au même titre que les autres animaux. Mais il ne peut même pas se consoler par la certitude de l'éternité de son espèce. En effet, on ne peut affirmer que les espèces des animaux dits "parfaits» qui, pour l'anonyme, ne peuvent se reproduire que par la seule semence, sont éternelles, alors qu'elles peuvent être anéanties par des cataclysmes (même s'il est peu probable qu'il ne puisse rester quelques rescapés pour perpétuer l'espèce), à la différence des plantes (comme on le voit dans le livre de Job) et des animaux imparfaits qui réapparaîtront toujours par génération spontanée. Ainsi, l'homme, qui "se vante d'être un animal parfait», est-il sous le rapport de l'éternité, inférieur à l'insecte lui-même, qui naît spontanément des éléments naturels et de la corruption des autres êtres vivants:

Les insectes, pour être engendrés, ont seulement besoin du soleil, de la terre et du limon de la terre, soit de pourriture, soit d'une autre matière indélébile: comme ces choses sont éternelles, les espèces des animaux qui naissent d'elle, elles aussi sont nécessairement éternelles ${ }^{11}$.

IO. IV, 4, p. 664.

II. II, 1, p. 178. 
Il s'agit expressément de rabattre la superbe humaine, de dépouiller l'homme de ses imaginaires privilèges ${ }^{12}$, ce qui est l'un des motifs clés de la philosophie de l'anonyme, héritée tout à la fois de Montaigne, de Charron et de Vanini. C'est aussi là un paradoxe, au moins apparent; l'homme aliéné dans la religion, soumis aux dieux qu'il invente lui-même, se donne pourtant dans la création le meilleur rôle, à la fois de centre, de noyau microcosmique et d'animal supérieur seul détenteur d'une âme rationnelle et intellective. Une fois les dieux révoqués, une fois l'âme ramenée à la condition mortelle et corruptible du corps, l'homme perd toute espèce de supériorité et de prérogative; il devient un animal parmi les autres, et un animal, sous bien des rapports, inférieurs aux autres, à la fois sur le plan de ses caractéristiques proprement corporelles - par sa fragilité, sa faiblesse, la précarité même de son espèce comme on vient de le voir -, et du point de vue de ses capacités intellectuelles, inférieures à bien d'autres animaux, affligé surtout d'une confusion et d'une anarchie des facultés qui le conduisent à inféoder sa raison à l'imagination et aux passions. Telle est la source de sa crédulité, qui le conduit à adopter des comportements allant à l'encontre de l'ordre de la nature; errance et erreurs dont les animaux sont parfaitement exempts.

L'anonyme connaît la littérature, assez abondante, qui envisage la possibilité d'une génération spontanée de l'homme à partir de la pourriture, c'est-à-dire de la corruption d'autres espèces sous l'action de la chaleur et de l'humidité ${ }^{13}$. Il sait aussi que certains en ont tiré des

I2. «Et si, en elles, une certaine durée éternelle devait exister, on devrait plutôt la repérer assurément dans ces choses que nous appelons les insectes, que dans celles que nous appelons parfaites, puisque celles-là sont engendrées de la matière permanente et éternelle, à savoir le soleil et la terre; les autres sont, en vérité, engendrées d'une semence, chacune, évidemment, selon son espèce. Et si elles semblent perdurer jusqu'à maintenant, il est cependant parfaitement possible qu'elles meurent. En cela, raisonnablement, il est nécessaire que l'audacieux orgueil de l'homme se précipite dans la profondeur de l'humilité: lui-même l'animal le plus stupide de tous, il se hausse, trop gonflé de morgue, au dessus des autres, alors qu'il devrait complètement se rabaisser au dessous de ceux qu'il pense être les plus vils. Cet homme, en effet, qui se vante d'être l'animal parfait, n'est pas si certain de l'éternité et de la durée de son espèce que de celle de ces animaux que l'on appelle des insectes" (II, 1, p. 177).

13. Il renvoie à Diodore de Sicile (Bibliothèque historique, I, 6-7); Avicenne (Sur le Déluge), Pic de la Mirandole, (900 conclusions philosophiques, cabalistiques et théologiques), Cardan (plutôt opposé d'ailleurs à la thèse de la possibilité d'une génération spontanée pour les animaux parfaits) et Vanini (De Admirandis, III, 37). Voir sur toutes ces références, l'appareil de notes de C. Canziani et G. Paganini 
sarcasmes visant une fois encore à avilir l'animal superbe, comme Vanini, lorsque, dans le De Admirandis ("Dialogue 37 », auquel l'anonyme ne manque pas de renvoyer) un athée de rencontre déclare que l'homme est né du mélange de cadavres de porc, de singes et de grenouille. Le créateur divin ne serait donc pas à l'origine de l'homme, mais des animaux en putréfaction, choisis sans aucun doute pour les vices qu'ils allégorisent.

Cependant, l'anonyme affirme son désaccord avec une telle hypothèse, par souci de rigueur scientifique:

Jamais je ne concéderai qu'un homme ou un autre de ces animaux qu'on appelle parfaits, puisse naître de la pourriture et du limon de la terre, ou qu'il en soit jamais né, quoi que veuille cette croyance qui s'est le plus fortifiée chez beaucoup ${ }^{14}$.

Mais cette impossibilité de la génération spontanée ne fait nullement de l'homme un être supérieur, au contraire, elle rend son espèce vulnérable. Prise dans les révolutions astrales qui dictent le retour des grandes catastrophes naturelles (déluges, etc.), l'espèce humaine est menacée, de manière récurrente, par le risque de l'extinction.

\section{La religion: générée, donc corruptible}

L'histoire humaine, dégagée de tout providentialisme, est entièrement prise dans le cours cyclique des révolutions naturelles; ainsi l'ensemble de ce que l'on considère aujourd'hui comme constituant la "culture" relève-t-il, pour l'anonyme, de la nature ${ }^{15}$. Dans l'histoire des sociétés humaines, tout ce qui apparaît, exactement comme tout ce qui naît dans la nature, est amené à se corrompre et à mourir. Le cycle des générations et des corruptions est dans ces domaines, non pas analogue, mais bien homologue à celui des êtres vivants générés "spontanément» (en fait «nécessairement») par l'action des éléments naturels et des astres. L'astrologie, science de l'action des astres sur les êtres produits à la

dans leur édition, p. 180 sq. et l'article de Fr. de Graux, «La génération spontanée de l'homme».

I4. II, 1, p. 178.

I5. Voir G. Paganini, «Temps et histoire dans la pensée libertine». 
surface de la terre, est ainsi donnée comme une clé privilégiée pour l'intelligibilité des vicissitudes des sociétés humaines.

C'est dans cette perspective que l'anonyme reprend explicitement au Pomponazzi des Incantations, en le citant très abondamment, son horoscope des religions. Dans leur naissance, leur développement, leur déclin et leur disparition, les religions obéissent à un déterminisme astral dont les principes et les lois sont accessibles à la connaissance humaine. Ainsi Pomponazzi, dans les lignes copiées par l'anonyme, s'exprimet-il au sujet des oracles du paganisme, modèle pour comprendre les "miracles", réductibles à des phénomènes naturels exceptionnels, qui semblent avoir abondé aux premiers temps du christianisme:

Les oracles, autrefois, ayant commencé, ils ont aussi pris fin et devaient prendre fin: tout individu, en effet, est engendré et corruptible; si quelque chose se corrompt, autre chose est engendré à partir du corrompu et s'oppose à lui, car leurs dispositions sont contraires. C'est pourquoi, comme les dieux antiques devaient disparaître, puisqu'ils avaient commencé, les dispositions et les usages de ces dieux devaient être corrompus par la génération et l'usage d'autres dieux, comme par l'introduction de la disposition à engendrer un homme le sang menstruel se corrompt ${ }^{16}$.

La comparaison à laquelle se livre Pomponazzi, d'une religion finissante au sang menstruel, purgé par la formation d'une "semence» nouvelle, selon le schéma aristotélicien et hippocratique, n'est évidemment pas anodine. Penser l'enchaînement des religions, tout au long de l'histoire humaine, sur le modèle de la génération et de la corruption des animaux comportait certainement une audace inouïe.

Dans ces pages citées dans le Theophrastus, Pomponazzi explique également que chaque religion ou "loi », selon le vocabulaire averroïste, obéit à un principe en fonction duquel «certains mots, certaines mœurs, certaines signes sont tenus en grand compte et en grand honneur». La loi suivante introduit un autre principe, contraire au précédent, en vertu duquel tout ce qui était honoré est désormais vilipendé et méprisé; ainsi, "à l'époque des idolâtres, il n'y avait rien de plus méprisable que la croix; mais à l'époque de la loi suivante, rien n'est plus en honneur que la

16. De Incantationibus, chap. XII (cité en III, 4, p. 405 sq.). 
croix elle-même» ${ }^{17}$. Ces changements de "lois» et par là de "principes" d'organisation de tout ce qui constitue une religion obéissent, non à un quelconque dessein providentiel, mais aux changements des conjonctures astrales, qui permettent de rendre raison, de manière strictement naturelle, des prodiges et "miracles" accomplis par les fondateurs, mais aussi de leurs disparitions, par succession des temps, lorsque les religions perdent de leur force et déclinent inexorablement. Telle est, précisément, la situation présente de la religion chrétienne, parvenue indubitablement à un stade de corruption avancé:

C'est pourquoi, maintenant aussi, dans notre foi, tout se refroidit, les miracles cessent, s'ils ne sont pas inventés et simulés: car la fin semble proche ${ }^{18}$.

Ce motif de la corruption présente de la religion chrétienne, où la simulation généralisée a pris la place des miracles authentiques, n'est certes pas original au siècle de la Réforme, mais Pomponazzi et l'anonyme auteur du Theophrastus à sa suite produisent une argumentation naturaliste visant à montrer que l'espoir d'une régénération interne du christianisme, qui se traduit par la prolifération des hérésies, est purement illusoire, ou plutôt est-il un signe supplémentaire d'une irréductible corruption.

\section{Fatalité de la religion et liberté du sage}

Le sage athée, promis à la persécution lorsqu'il est découvert (d'où le rigoureux anonymat), trouve sans doute une certaine revanche à ce spectacle du délitement de cette religion parmi les plus contraignantes et les plus cruelles pour la nature. Mais son naturalisme l'oblige à ne nourrir aucune illusion, au sens où, précisément, le sage sait que l'illusion religieuse obéit en fin de compte à une nécessité naturelle inscrite en l'homme, et qu'une "loi" succédera fatalement à une autre, comme l'exige l'enchaînement cyclique des choses humaines soumises aux transformations astrales. En d'autres termes, la corruption du christianisme est inséparable de la gestation d'une religion nouvelle et ne peut donc

I7. III, 4, p. 407.

I8. Ibid. 
être interprétée comme la promesse d'une libération de l'humanité des illusions religieuses. Nous sommes, avec le Theophrastus, aux antipodes d'une eschatologie de l'athéisme. C'est là tout ce qui sépare l'irréligion et l'athéisme dits «libertins» de ce qu'il est désormais convenu d'appeler les Lumières radicales.

Pourtant, cet affranchissement est immédiatement possible pour le sage lui-même, comme il l'a du reste toujours été, ainsi que le montre la très longue généalogie des philosophes athées qui, dans toutes les religions, se sont libérés de l'illusion religieuse en prenant la nature pour guide. C'est à ce point que le naturalisme intégral de l'anonyme subit une inflexion notable et révèle une aporie qu'il est bien difficile de ne pas appréhender comme une contradiction: les religions qui se succèdent dans l'histoire selon le cycle des générations et des corruptions possèdent des causes strictement naturelles et pourtant elles détournent les hommes de vivre selon la nature, d'évoluer dans la vérité, l'égalité et la liberté naturelles, comme le font les autres animaux; elles sont une puissance d'erreur, d'injustice et d'asservissement (toutes choses «contrenature") dans les mains de ceux qui y trouvent leur intérêt; princes, prêtres et parfois même philosophes. Les sociétés humaines, telles que nous les connaissons, où le mensonge religieux sert d'abord à fonder et légitimer la propriété privée et l'inégalité entre les hommes, l'asservissement des uns aux autres, à travers les mécanismes de la superstition, représentent sans nul doute, pour l'auteur du Theophrastus, une forme de vie dévoyée, dégradée et pour tout dire corrompue. La civilisation est en effet comprise comme une corruption de l'état de nature. Le sage seul, affranchi des mensonges de la religion et qui prend comme guide la seule loi de nature, échappe à cet état de corruption généralisée.

L'anonyme n'utilise guère le terme de corruptio en ce sens ${ }^{19}$, et cela se comprend, puisqu'il a rejeté le vocabulaire chrétien de la chute, et l'idée d'une corruption ontologique de la nature (en l'homme et hors de l'homme par le péché). Mais comment qualifier l'état de dégénérescence actuel, déjà d'ailleurs fort ancien, des société humaines par rapport à l'état de nature, sinon en s'appropriant le vocabulaire moral de la transgression, de la faute, de la déviance, de la perversité?

I9. Voir cependant le passage cité infra au sujet du «jugement du peuple perverti et corrompu». 


\section{La civilisation comme corruption de la nature}

Le fond de la religion, en tant qu'il relève de la nature, n'est pas erroné ni pervers. Il n'est en effet rien d'autre d'une part que la reconnaissance de l'importance déterminante des astres dans le cours des choses naturelles et donc humaines (l'anonyme insiste beaucoup sur le fait que les premières religions dont nous avons gardé souvenir sont les religions astrales, suggérées par le terme même de theos ${ }^{20}$ ) et d'autre part l'obéissance, que l'on retrouve dans toutes les religions, aux préceptes de la loi naturelle. En effet, pour mensongères et perverses, pour corrompues qu'elles soient, les religions n'ont pu étouffer complètement les commandements de la nature; il faudrait pour cela arracher l'homme à la nature, ce qui n'est évidemment pas possible. Les lois primordiales de la vie sociale, auxquelles leurs auteurs attribuent mensongèrement une origine divine, à savoir "n'offense personne", "aime ton prochain comme toi-même", "ne fais pas à autrui ce que tu ne veux pas qu'on te fasse ", sont des lois instaurées non par des hommes ou des dieux, mais par la nature même, et elles sont intimement liées à [la] loi [...] de la conservation de l'animal ${ }^{21}$.

La loi fondamentale est en effet celle de la "conservation", au nom de laquelle toute action est bonne et légitime. On voit en fait comment la question de la théodicée, une fois les dieux révoqués, reflue sur la nature: aussi l'anonyme a-t-il le souci de développer ce que l'on pourrait nommer une "physidicée». La nature ne connaît pas le mal, elle ne connaît pas le péché et donc la corruption morale: «il n'y a par nature pas de mal, rien qui soit illicite, inconvenant». Ainsi tous les actes présentés par les morales religieuses et les lois positives comme honteux ou criminels, signes d'une nature dépravée ou corrompue, ne le sont en fait pas: ni le vol, ni l'adultère, ni la sodomie, ni le sacrilège, pas même l'inceste ou l'anthropophagie ne sont en eux-mêmes de mauvaises actions ${ }^{22}$ :

20. Selon l'auteur, théos signifie d'abord cursor, coureur, et désignait les astres: «Ce nom a d'abord été appliqué au soleil, à la lune et aux autres corps célestes»; ce nom "leur a été imposé par la faculté de courir et d'errer à travers l'espace du ciel, faculté accordée par la nature de toute éternité» (I, 6, p. 158).

2I. VI, 1, p. 786, trad. H. Bah-Ostrowiecki, p. 223.

22. VI, 1, p. 794 et sq., trad. H. Bah-Ostrowiecki, p. 234-237. 
Seules les lois et les coutumes humaines donnent une définition et un statut au mal, alors que par nature toute chose est bonne, voire excellente ${ }^{23}$.

Cela ne veut pas dire que tous ces actes doivent être accomplis, car beaucoup d'entre eux, selon les circonstances, sont accompagnés d'inconvénients multiples, en partie, mais pas seulement, du fait de l'existence des lois humaines répressives. En effet, la condition du bonheur n'est pas seulement de suivre les impulsions de la nature, mais aussi d'échapper aux troubles et aux désagréments de toutes sortes. C'est du reste obéir à la nature qui nous commande de fuir la douleur et le trouble pour autant que cela est possible (l'influence épicurienne est ici manifeste). Aussi la sagesse commande-t-elle de nous retenir de tous les actes susceptibles de corrompre notre félicité ${ }^{24}$.

Sur cette base, extrêmement cohérente, il apparaît que le mal n'est autre chose que "l'opinion» que nous en avons, "l'opinion du mal», la "plus vaines des choses", inscrite dans les coutumes, les préceptes de la religion et les lois positives. Ce mal là, n'est cependant pas illusoire mais véritable, qui consiste d'abord en la prétention de se dresser contre la nature et voue ainsi l'humanité au malheur. Il existe bien en l'homme un agent de corruption, au sens moral, politique mais d'abord ontologique, qui le fait déchoir de l'état de nature et le condamne au malheur de la civilisation. Cet agent reçoit dans le texte de l'anonyme le nom d'«opinion», héritier en cela des philosophies grecques et latines de la nature qui faisaient de la doxa ou opinion, la faculté dénaturante en l'homme, puissance d'erreur et inconduite. L'opinion est, dans le Theophrastus, tout à la fois faux savoir, préjugé, superstition, présomption de soi et de l'humanité eu égard à la nature. L'opinion s'oppose à la «raison ", qui est la voix de la nature, et qui n'est d'ailleurs rien d'autre que ce que l'on appelle l'instinct, dont les animaux sont aussi bien pourvus, et parfois mieux, que les hommes ${ }^{25}$.

L'opinion sépare l'homme de sa condition naturelle et, ce faisant, engendre tous les "monstres" (terme récurrent dans le texte associé au mot d'opinion) qui affligent l'homme: la conviction de la supériorité de

23. VI, 1, p. 800, trad. H. Bah-Ostrowiecki, p. 240.

24. VI, 1, p. 802, trad. H. Bah-Ostrowiecki, p. 241.

25. VI, 2, p. 823, trad. H. Bah-Ostrowiecki, p. 270. 
l'homme sur les autres êtres naturels ${ }^{26}$, l'instauration de la domination des uns sur les autres, la séparation du tien et du mien, les mensonges des religions, l'invention des lois, des arts et des sciences. On peut à cet égard parler de corruption systématique de la nature, qui advient d'abord par l'abandon de l'égalité et de la liberté naturelles. Le moteur de celle-ci réside dans les passions humaines nourries par l'opinion, selon un clivage anthropologique constitutif de l'état de civilisation. Les uns sont portés en effet par l'ambition et la présomption, l'outrecuidance ou vanité, opinion d'une supériorité imaginaire. Ils recourent à la fois à la violence pour asservir autrui, et à la tromperie; la force ne suffisant jamais à asseoir la domination, le mensonge y est en effet nécessaire et le mensonge par excellence est celui de la religion, par lequel les dieux sont inventés pour maintenir les peuples en obéissance et servitude. Les autres se caractérisent par leur crédulité et leur imbécillité, qui se laissent mener par la crainte des châtiments et l'espoir des récompenses, en cette vie-ci, mais surtout en l'au-delà. Ce second type d'hommes est celui que le Theophrastus nomme "peuple", qui est entièrement enfermé dans le carcan de l'opinion, esclave de la superstition, ce qui n'est pas le cas de leurs maitres (princes, magistrats et prêtres) qui fabriquent et entretiennent les mensonges religieux et, de ce fait, gardent au moins une distance critique envers les opinions qu'ils forgent et qu'ils exploitent à leur profit. Le jugement du peuple est, dès le berceau, "perverti et corrompu»

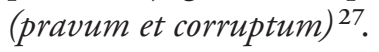

Tout ce qui compose la civilisation, «les institutions et les règlements, les livres, les sciences et les connaissances, les arts, les collèges, les couvents, les officines en tout genre; et là on travaille jour et nuit depuis le berceau, à ce que la nature soit complètement pervertie ou du moins violemment torturée par l'éducation ${ }^{28}$. La corruption systématique de la nature, en laquelle consiste l'état de civilisation, est ainsi un travail, un exercice permanent où se conjoignent la production instituée des savoirs

26. «Les hommes ont mis un comble à la confusion qui les a saisis quand ils se sont placés au-dessus du reste des êtres animés, comme si [la nature] avait produit un élément supérieur à un autre, dans l'intention d'instaurer un maître au sein du règne animal», VI, 1, p. 803, trad. H. Bah-Ostrowiecki, p. 245.

27. VI, 4, p. 883, trad. H. Bah-Ostrowiecki, p. 347. Voir L. Bianchi, «Sapiente e popolo nel Theophrastus redivivus".

28. VI, 3, p. 859, trad. H. Bah-Ostrowiecki, p. 310 sq. 
fallacieux et une discipline constante sur les esprits et les corps, afin de les contraindre, par la crainte et l'espérance, à brider leurs désirs naturels.

Le Theophrastus décrit ainsi un cercle vicieux entre l'opinion, par définition anti ou contre naturelle (si une opinion était conforme à la nature elle ne serait autre chose qu'un droit usage de la raison ou de l'instinct), et les lois qu'elle permet d'instituer, mais qui, à leur tour, sont la source et même "la cause efficiente» des monstrueuses opinions embrassées par les peuples:

[...] qui voudra bien examiner et peser scrupuleusement les moyens par lesquels les lois, causes productrices de toutes les opinions, ont progressé et pris naissance parmi les hommes, n'aura pas de mal à percer à jour l'erreur dont ils sont le jouet et à connaître l'inanité des lois. Toutes les lois ont pour principe et origine des fables et des fictions: de fait, comme les premiers princes se rendaient compte que l'espoir et la crainte étaient les moyens les plus efficaces de gouverner la foule, ils introduisaient les religions et leurs mensonges, qui reposent entièrement sur ces deux affections... ${ }^{29}$.

En même temps que l'opinion, qui est l'asservissement de la raison aux passions et affections, en particulier à travers la croyance aux mensonges de la religion, est ainsi mise en cause la "ruse» (calliditas) par laquelle les législateurs, prêtres et philosophes, forgent et entretiennent les fables religieuses. L'habileté et la finesse d'esprit dont l'homme s'enorgueillit tant, comme l'explique un passage de la Nature des dieux de Cicéron repris ponctuellement dans le Theophrastus, sont en fait les marques de la faiblesse de la raison humaine. "Quel plus grand don la nature leur auraitelle fait, si elle avait voulu leur nuire?», demande Cicéron. L'anonyme renchérit en remarquant que la «raison humaine produit de si grandes vanités et de si grandes frivolités qu'elle a été donnée pour conduire aux inepties et aux fables» ${ }^{30}$. Ainsi la raison humaine, dans la ruse employée à tromper autrui par les fables de la religion, comme dans l'opinion sollicitée à la fois pour les inventer et les croire, est-elle en fait une raison défaillante, corrompue, si on la compare à la raison - ou instinct - des animaux.

29. VI, 3, p. 862-863, trad. H. Bah-Ostrowiecki, p. 314 sq. 30. VI, 3, p. 877, trad. H. Bah-Ostrowiecki, p. 338. 


\section{Une pensée athée de la vanité}

Mais, finalement, à qui la faute, sinon à la nature elle-même, qui a donné à l'homme cette raison frivole, encline à forger des fables, à opiner à des représentations mensongères, à seconder des passions déréglées? Ce dérèglement lui-même, contre nature, trouve bien sa source dans la nature.

Il ne saurait en être autrement, même si, dans le cadre de sa "physidicée», l'anonyme exclut de faire porter une quelconque faute sur la nature. L'homme ne serait-il pas un être intrinsèquement prédisposé à la corruption ou, si l'on veut, naturellement disposé à la dénaturation? Mais il faut se souvenir qu'en dehors de la nature, il n'y a rien; la dénaturation humaine, sa prétention à s'élever au-dessus de la nature, à se feindre une âme immortelle ${ }^{31}$, etc. ne sont que vanité, inanité, ineptie, imbécillité, ignorance.

Et en effet, l'anonyme débouche sur les thèmes de l'histrionisme universel («le monde entier joue la comédie») et, une fois encore, de la vanité de l'homme:

Une bonne compréhension de tout ce que nous avons dit de la vanité humaine dans les pages précédentes fera sans aucun doute estimer que la substance des choses humaines est dépourvue de toute perfection, pleine de désordres, et de plus pétrie de crainte et d'espoir, choses dans lesquelles il ne peut y avoir rien de fiable, de sûr, de solide; pour tout dire une ombre, et le songe d'une ombre ${ }^{32}$.

Il s'agit là, avec la métaphore de Pindare (l'homme est le «songe d'une ombre»), de la plus commune expression du motif de la vanité de l'homme, ici rigoureusement déchristianisé. L'homme n'est en effet pas un néant devant Dieu, mais dans la nature, non pas tant du fait de la fragilité et fugacité de ses jours qu’à cause de ses défauts, de ses défaillances intellectuelles et morales qui lui font imaginer des choses aussi ineptes que la divinité et l'immortalité de l'âme.

3I. "Les fictions des Enfers et du paradis sont pour lui [l'homme] de tels motifs de s'enorgueillir, de se persuader que la raison le rend supérieur aux autres animaux, parce qu'il s'est forgé l'idée qu'il a une âme immortelle» (VI, 3, p. 877, trad. H. BahOstrowiecki, p. 338).

32. Ibid. 
Ce motif de la vanité avait déjà été développé, dans une perspective sceptique, par Corneille Agrippa et surtout par Charron, qui l'avait fait ainsi pénétrer profondément dans la littérature dite "libertine» ${ }^{33}$. Nous en trouvons une version radicale dans les vers de Jacques Vallée Des Barreaux qui déplore le néant de l'homme et surtout la misère dans laquelle le plonge la nature, non pas tant en le faisant mortel, mais en lui donnant la raison, qui lui instille le poison de la conscience de ses malheurs, de sa précarité et de la fin imminente. L'homme apparaît ainsi, par la faute d'une nature contre laquelle le poète jette ses imprécations, comme un animal malheureux, un animal déchu, corrompu du fait même de la possession de la raison, faculté maudite et méprisable:

Mortels, qui vous croyez, quand vous venez à naistre,

Obligez à Nature, ô quelle trahison!

Se montrer un moment, pour jamais disparoistre.

Et pendant que l'on est, voir des maux à foison.

Tenant plus du néant que l'on ne fait de l'être,

Je l'ay dit autrefois et bien moins en saison,

Estudions-nous plus à joüir qu'à connoistre,

Et nous servons des sens plus que de la raison.

D’un sommeil éternel ma mort sera suivie,

J'entre dans le néant quand je sors de la vie,

O desplorable estat de ma condition!

Je renonce au bon sens, je hay l'intelligence,

D'autant plus que l'esprit s'élève en connoissance,

Mieux voit-il le sujet de son affliction ${ }^{34}$.

La position de Des Barreaux, misologique, foncièrement pessimiste, accusant la nature d'avoir fait l'homme tel qu'il est, vain et malheureux, mérite d'être citée dans le contexte d'une lecture du Theophrastus. En

33. Agrippa, De l'incertitude, vanité, \& abus des sciences. Sur le lien entre ces pages du Theophrastus et La Sagesse de Charron, voir l'édition citée de la VI e partie du Theophrastus, p. 1541, n. 1 de la p. 338.

34. Sonnet Qui multiplicat intellectum multiplicat afflictionem, dans Des Barreaux, Euvres complètes, p. 272. Voir également le sonnet: Qui addit scientiam addit et laborem: "[...] Peu de bon sens, point de sçavoir,/ Foutre de la philosophie./ Je me dégrade de raison,/ je veux devenir un oison,/ Et me sauver dans l'ignorance" (ibid., p. 273). 
effet, tout en exploitant le même motif de la vanité sans Dieu, l'anonyme, loin de dénoncer la raison comme telle et de s'en prendre à la nature, s'emploie à montrer que la méditation sur la vanité relève au contraire du bon usage de la raison, qui ramène l'homme à la nature et lui donne accès au bonheur et à la sagesse:

[...] il nous faut toujours avoir l'esprit occupé par la mort, afin de mieux dépouiller tout souci de l'avenir; et nous devons continuellement nous réjouir, comme si nous étions continuellement à la veille de notre mort. Telle est l'observation la plus consommée de la loi naturelle, et la félicité parfaite ${ }^{35}$.

Le discours sur la vanité, le memento mori du sage affranchi des fables d'arrières mondes, pour parler comme Nietzsche, est ici exercice de mépris de la mort (tout le livre $\mathrm{V}$ du traité porte d'ailleurs le titre $D e$ contemnenda morte: «De la mort devant être méprisée»).

Par la contemplation de la caducité et de la mort, envisagée sans crainte comme le processus normal de la corruption de toutes les choses de la nature en ce "bas» monde (sublunaire), la raison se défait des opinions mensongères, "foule les lois à ses pieds" (une autre expression récurrente du traité), revient à la loi naturelle et assure le bonheur du sage. C'est d'ailleurs exactement sur ce motif que s'achève l'ouvrage ${ }^{36}$. Il existe une voie courte, ouverte à tous les esprit forts, qui conduit de la pleine conscience de la vanité (avec tout ce qu'elle implique, à commencer par la conscience de l'ineptie de la religion qui exploite le même motif pour son propre compte) au bonheur par l'adoption d'une vie conforme aux seuls enseignements de la nature.

Cette conversion à la nature s'accompagne de l'impératif absolu, une fois encore réitéré, de se séparer du "peuple» au "jugement perverti et corrompu» et, pour cette raison, pervers et corrupteur. Apparaît alors toute l'importance que joue dans l'éthique du Theophrastus l'anthropologie clivée d'ascendance averroïste, selon laquelle il existe

35. VI, 4, p. 883, trad. H. Bah-Ostrowiecki, p. 347.

36. L'anonyme cite longuement le chapitre II du livre biblique de la Sagesse: «Il n'est personne, que l'on sache, qui soit revenu des Enfers, parce que nous sommes nés du néant et qu’après nous serons comme si nous n’avions pas été» (VI, 4, p. 926, trad. H. Bah-Ostrowiecki, p. 403). 
des différences naturelles radicales d'homme à homme, du même ordre que celles qui sépare un homme peint d'un homme véritable ${ }^{37}$. La plus grande partie de l'humanité semble incapable de faire un droit usage de la raison et de se débarrasser donc des fictions de l'opinion. Ce qui est accessible au sage ne l'est pas à cette humanité déficiente et c'est pourquoi, du reste, la religion et les lois sont nécessaires qui, pourtant ne rendent pas le "peuple» meilleur (bien au contraire! ${ }^{38}$ ), mais permettent de maintenir malgré tout la vie sociable.

Pour l'anonyme, et c'est une représentation qu'il partage avec la plupart des auteurs que l'on range parmi les libertins érudits (Charron, Naudé, La Mothe le Vayer, etc.), il est une partie de l'humanité, la plus grande part, qui - au moins en l'état actuel des choses - est incapable de sagesse, irrémédiablement corrompue et corruptrice: le "peuple». Il nous semble qu'ici le préjugé social, derrière le motif anthropologique, pèse de tout son poids. Car l'anonyme, comme les auteurs dont nous venons de citer les noms, semble souvent admettre que le "peuple», du fait de sa constitution propre, ne saurait vivre dans l'égalité et la liberté naturelles.

Tout à la fois, l'auteur considère que l'état de nature, d'égalité, de liberté et de communauté, n'est pas une fiction mais une réalité historique, confortée par l'existence de "peuples", aujourd'hui encore, qui en sont restés au plus proche ${ }^{39}$. Il ne remet d'ailleurs jamais directement en question l'affirmation de l'égalité naturelle des hommes, ou plus exactement de leur égalité dans l'état de nature, où le fait d'être plus ou moins subtil n'a aucune importance. Par contre, cette différence joue un rôle primordial dans l'instauration et la conservation de l'état damné de civilisation. Certes, les plus subtils, par leur désir de domination et leurs ruses mensongères et malveillantes, trahissent autant, sinon plus, la loi de nature que ces esprits faibles, inexpérimentés et crédules qu'ils se soumettent par la violence et le mensonge. Pourtant ce sont

37. "Il faut penser que les hommes diffèrent des hommes eux-mêmes; en effet, innombrables sont ceux qui sont bien plus habiles et plus ingénieux que les autres" (VI, 3, p. 861, trad. H. Bah-Ostrowiecki, p. 311). Voir L. Bianchi, «Filosofi, uomini e bruti, note per la storia di un'antropologia averroista» et M. A. Granada, "Note sull'averroismo di Giordano Bruno».

38. "Aucun dieu, aucune religion, ni aucune autre loi quelle qu'elle soit n'a jamais rendu les hommes meilleurs» (VI, 3, p. 864, trad. H. Bah-Ostrowiecki, p. 316).

39. Voir au sujet des peuples américains avant la colonisation, VI, 3, p. 869, trad. H. Bah-Ostrowiecki, p. 326. 
bien les premiers, parce qu'ils ne sont jamais complètement dupes des fables qu'ils inventent ou entretiennent, qui sont susceptibles de pousser jusquau bout la critique et embrasser la vie naturelle; on trouve du reste parmi eux le précepteur d'Alexandre, de grands magistrats comme Cicéron, des conseillers d'Etat comme Sénèque, voire des empereurs comme Marc Aurèle. Certes, on en trouve aussi qui ont toujours fui la vie publique, comme Socrate ou Diogène le cynique; et, comme Le Vayer et la plupart des autres auteurs d'une conception élitaire de la sagesse déniaisée, l'anonyme se refuse à donner une définition sociale de ce qu'il entend par peuple. Pourtant, spontanément, il conçoit son aréopage de sages comme tiré, en quelque sorte prélevé, de l'élite sociale.

Ce sont ces préjugés sociaux, d'autant plus puissants qu'ils ne sont pas assumés, qui lui font concevoir le peuple ignorant et, dirait-on après Marx, aliéné, comme le lieu par excellence de la déchéance et de la corruption de l'humanité. Parce que cette corruption n'est pas seulement due à l'éducation, à l'inculcation de l'erreur et de la fausseté, mais aussi à une faiblesse anthropologique intrinsèque, relevant proprement de la nature, l'auteur du Theophrastus est fermé à toute idée d'une régénération possible de l'humanité guidée par les philosophes; ou plutôt faut-il dire que cette idée ne l'effleure même pas. Par contre, sa conception physiciste du cycle ininterrompu des générations et des corruptions entretient chez lui la conviction selon laquelle, naturellement, l'organisation présente du monde humain prendra fin et qu'une autre commencera après elle, radicalement différente et en rupture avec la précédente. Ce monde d'ailleurs, vu l'image absolument négative que l'anonyme dresse de la société chrétienne tardive, ne pourra être que meilleur. Son naturalisme intégral (du moins sur le plan des principes) laisse place à l'idée que ces hommes alors pourraient être d'une tout autre trempe, aussi jaloux de leur liberté et de leur égalité natives que l'ont été leurs très lointains ancêtres, dans ce passé lointain dont l'humanité a gardé la mémoire sous le nom d'âge d'or.

Jean-Pierre Cavaillé EHESS, Paris 


\section{BIBLIOGRAPHIE}

\section{Textes}

Agrippa, Corneille, De l'incertitude, vanité, \& abus des sciences, Genève, P. Chou, 1630.

[Anonyme], Theophrastus redivivus, a cura di Guido Canziani e Giani Paganini, Firenze, La Nuova Italia, 1981-1982, 2 vol.

Bah-Ostrowiecki, Hélène, "Theophrastus redivivus. Sixième traité, traduction du latin", introduction et notes, in Libertins du XVII siècle, sous la direction de Jacques Prévot, vol. 2, Paris, Gallimard, La Pléiade, 2004, p. 217-404.

Des Barreaux, Jacques Vallée, Euvres complètes, éd. par MarieFrançoise Baverel-Croissant, Paris, Honoré Champion, 2001.

\section{Travaux}

Bah-Ostrowiecki, Hélène, Le Theophrastus redivivus. Erudition et combat antireligieux au XVII siècle, Paris, Honoré Champion, 2012.

Bianchi, Laurenzo, "Sapiente e popolo nel Theophrastus redivivus», Studi Storici, 24 (1983), p. 137-164.

Bianchi, Luca, "Filosofi, Uomini e bruti, note per la storia di un'antropologia averroista", Rinascimento, 32 (1992), p. 185-201.

Gengoux, Nicole, Le "Theophrastus redivivus» ou l'athéisme comme position philosophique à l'Age classique, thèse de doctorat, Université Paris I, 2008, publiée sous le titre: Un athéisme philosophique à l'Age classique: le "Theophrastus redivivus», 1659, Paris, Honoré Champion, 2014. 
Gengoux, Nicole, Moreau, Pierre-François (dir.), Entre la Renaissance et les Lumières: le Theophrastus redivivus (1659), Paris, Honoré Champion 2014.

Granada, Miguel Angel, "Note sull'averroismo di Giordano Bruno", Giornale critico della filosofia italiana, 71 (1992), p. 248-275.

Graux, François de, "La génération spontanée de l'homme", in Révolution scientifique et libertinage, éd. par Alain Mothu, Bruxelles, Brepols, 2000, p. 177-192.

Gregory, Tullio, Theophrastus redivivus. Erudizione e ateismo nel Seicento, Naples, Morano, 1979.

Paganini, Gianni, «Temps et histoire dans la pensée libertine», Archives de Philosophie, 49 (1986), p. 583-602.

Rodríguez Donis, Marcelino, Materialismo y Ateísmo. La Filosofía de un Libertino del XVII, Sevilla, Universidad de Sevilla, 2008. 
